\title{
ON THE MAXIMALITY OF SUMS OF NONLINEAR MONOTONE OPERATORS
}

\author{
BY \\ R. T. ROCKAFELLAR( ${ }^{(1)}$
}

1. Introduction. Let $X$ be a real Banach space and let $X^{*}$ be the dual of $X$, with $\left\langle x, x^{*}\right\rangle$ written in place of $x^{*}(x)$. A monotone operator from $X$ to $X^{*}$ is a (generally multivalued) mapping $T$ such that

$$
\left\langle x-y, x^{*}-y^{*}\right\rangle \geqq 0 \quad \text { whenever } x^{*} \in T(x), y^{*} \in T(y) .
$$

Such an operator $T$ is said to be maximal if its graph, i.e. the set

$$
G(T)=\left\{\left(x, x^{*}\right) \mid x \in X, x^{*} \in T(x)\right\} \subset X \times X^{*}
$$

is not properly contained in the graph of any other monotone operator $T^{\prime}: X \rightarrow X^{*}$. The set

$$
D(T)=\{x \in X \mid T(x) \neq \varnothing\}
$$

is called the effective domain of $T$, and $T$ is said to be locally bounded at a point $x \in D(T)$ if there exists a neighborhood $U$ of $x$ such that the set

$$
T(U)=\bigcup\{T(u) \mid u \in U\}
$$

is a bounded subset of $X$.

It is apparent that, given any two monotone operators $T_{1}$ and $T_{2}$ from $X$ to $X^{*}$, the operator $T_{1}+T_{2}$ is again monotone, where

$$
\begin{aligned}
\left(T_{1}+T_{2}\right)(x) & =T_{1}(x)+T_{2}(x) \\
& =\left\{x_{1}^{*}+x_{2}^{*} \mid x_{1}^{*} \in T_{1}(x), x_{2}^{*} \in T_{2}(x)\right\} .
\end{aligned}
$$

If $T_{1}$ and $T_{2}$ are maximal, it does not necessarily follow, however, that $T_{1}+T_{2}$ is maximal-some sort of condition is needed, since for example the graph of $T_{1}+T_{2}$ can even be empty (as happens when $D\left(T_{1}\right) \cap D\left(T_{2}\right)=\varnothing$ ).

The problem of determining conditions under which $T_{1}+T_{2}$ is maximal turns out to be of fundamental importance in the theory of monotone operators. Results in this direction have been proved by Lescarret [9] and Browder [5], [6], [7]. The strongest result which is known at present is:

THEOREM (BROWDER [6], [7]). Let $X$ be reflexive, and let $T_{1}$ and $T_{2}$ be monotone operators from $X$ to $X^{*}$. Suppose that $T_{1}$ is maximal, $D\left(T_{2}\right)=X, T_{2}$ is single-valued

Received by the editors September 24, 1968.

( ${ }^{1}$ This research was supported in part by the Air Force Office of Scientific Research under grant AF-AFOSR-1202-67. 
and hemicontinuous (i.e. continuous from line segments in $X$ to the weak ${ }^{*}$ topology in $X^{*}$ ), and $T_{2}$ carries bounded sets into bounded sets. Then $T_{1}+T_{2}$ is a maximal monotone operator.

The conditions here on $T_{2}$ imply in particular that $T_{2}$, like $T_{1}$, is maximal (Browder [3, Theorem 1.2]).

The purpose of this paper is to establish the following considerably more general result (where int and cl denote interior and (strong) closure, respectively).

THEOREM 1. Let $X$ be reflexive, and let $T_{1}$ and $T_{2}$ be maximal monotone operators from $X$ to $X^{*}$. Suppose that either one of the following conditions is satisfied:

(a) $D\left(T_{1}\right) \cap$ int $D\left(T_{2}\right) \neq \varnothing$, or

(b) there exists an $x \in \mathrm{cl} D\left(T_{1}\right) \cap \mathrm{cl} D\left(T_{2}\right)$ such that $T_{2}$ is locally bounded at $x$.

Then $T_{1}+T_{2}$ is a maximal monotone operator.

Conditions (a) and (b) of Theorem 1 are actually equivalent, as we have shown elsewhere [16, Theorem 1].

The derivation of Theorem 1 will rest heavily on the theory already developed by Browder in [6] and elsewhere. In the case where $T_{1}$ and $T_{2}$ are the subdifferentials of lower semicontinuous proper convex functions on $X$ [12], [13], Theorem 1 could be deduced, however, directly from Fenchel's duality theorem and related results [11].

When $X$ is finite-dimensional, Theorem 1 may be refined slightly in terms of relative interiors. (The relative interior of a subset $C$ of $X$, denoted by ri $C$, is the interior of $C$ relative to the affine hull of $C$, which is the intersection of all the hyperplanes containing $C$.)

THEOREM 2. Let $X$ be finite-dimensional, and let $T_{1}$ and $T_{2}$ be maximal operators from $X$ to $X^{*}$ such that

$$
\text { ri } D\left(T_{1}\right) \cap \text { ri } D\left(T_{2}\right) \neq \varnothing .
$$

One of the main motivations behind Theorems 1 and 2 is that such results make it possible, as Browder has remarked [6, p. 92], to derive theorems about "variational inequalities" from fundamental theorems about the ranges and effective domains of (multivalued) maximal monotone operators. Some applications of this sort will be considered in $\$ 4$. For the sake of applications to a class of variational inequalities studied in terms of hemicontinuity by Hartman-Stampacchia [8] and Browder [4], it will be useful to have, along with Theorems 1 and 2, the following special maximality theorem.

We shall say that an element $x^{*} \in X^{*}$ is normal to a convex subset $K$ of $X$ at a point $x$ if

$$
x \in K \text { and }\left\langle u-x, x^{*}\right\rangle \leqq 0, \quad \forall u \in K .
$$

For each $x \in X$, the set of all $x^{*}$ normal to $K$ at $x$ is classically called the normal 
cone to $K$ at $x$. The normal cone to $K$ at $x$ is a weak* closed convex cone in $X^{*}$, empty by definition when $x \notin K$, but containing at least the zero vector of $X^{*}$ (and hence nonempty) when $x \in K$. The multivalued mapping from $X$ to $X^{*}$ which assigns to each $x \in X$ the normal cone to $K$ at $x$ will be referred to as the normality operator for $K$. (This mapping is actually the subdifferential of the indicator of $K$, so that it is a maximal monotone operator with effective domain $K$, if $K$ is a nonempty closed convex set [12], [13].)

THEOREM 3. Let $K$ be a nonempty closed convex subset of $X$ (where $X$ is not necessarily reflexive), and let $T_{1}: X \rightarrow X^{*}$ be the normality operator for $K$. Let $T_{2}: X \rightarrow X^{*}$ be any single-valued monotone operator (not necessarily maximal) such that $D\left(T_{2}\right) \supset K$ and $T_{2}$ is hemicontinuous on $K$, i.e. continuous along each line segment in $K$ with respect to the weak ${ }^{*}$ topology of $X^{*}$. Then $T_{1}+T_{2}$ is a maximal monotone operator.

2. Preliminary results. In this section we shall only be concerned with the case where $X$ is reflexive. Asplund [1] has shown by means of a theorem of Lindenstrauss that, in this case, there exists an equivalent norm on $X$ which is everywhere Gâteaux differentiable except at the origin and whose polar norm on $X^{*}$ is everywhere Gâteaux differentiable except at the origin. (Under such a norm, the unit balls of $X$ and $X^{*}$ are strictly convex.) For notational simplicity, we may assume that the given norm on $X$ already has these special properties. We denote by $J$ the Gâteaux gradient of the function $h(x)=(1 / 2)\|x\|^{2}$. Thus $J$ is the duality mapping which assigns to each $x \in X$ the unique $J(x) \in X^{*}$ such that

$$
\langle x, J(x)\rangle=\|x\|^{2}=\|J(x)\|^{2} .
$$

(See [2].) As is known, $J$ maps $X$ one-to-one onto $X^{*}$ and is continuous from the strong topology to the weak* topology. Also, $J$ is a strictly monotone operator, i.e.

$$
\langle x-y, J(x)-J(y)\rangle>0 \quad \text { when } x \neq y \text {. }
$$

For any monotone operator $T$ from $X$ to $X^{*}$, we define the mapping $T^{-1}$ by

$$
T^{-1}\left(x^{*}\right)=\left\{x \mid x^{*} \in T(x)\right\} \text {. }
$$

It is obvious that $T^{-1}$ is a monotone operator from $X^{*}$ to $X$, and (assuming $X$ to be reflexive) $T^{-1}$ is maximal if and only if $T$ is maximal. We denote the range of $T$ by $R(T)$. Thus

$$
R(T)=D\left(T^{-1}\right)=\bigcup\{T(x) \mid x \in X\} .
$$

The main tool which we shall use in proving Theorem 1 is a generalization, essentially due to Browder [6], of the fundamental Hilbert space theorem of Minty [10].

Proposition 1. Let $X$ be reflexive, and let $J$ be the duality mapping defined above. Let $T: X \rightarrow X^{*}$ be any maximal monotone operator. Then, for any $\lambda>0, R(T+\lambda J)$ 
is all of $X^{*}$ and $(T+\lambda J)^{-1}$ is a single-valued maximal monotone operator from $X^{*}$ to $X$ which is demicontinuous, i.e. continuous from the strong topology to the weak topology.

Proof. If $0 \in D(T)$, this follows from the argument given by Browder at the beginning of the proof of Theorem 4 of [6], except that Lemma 10 of [6] should be invoked in place of Lemma 8. We want to use this result, however, even in the case where $0 \notin D(T)$, so we must give a somewhat modified proof. Let $a \in D(T)$, and let $T_{1}$ and $T_{2}$ be the monotone operators defined by

$$
T_{1}(x)=T(x+a), \quad T_{2}(x)=\lambda J(x+a) .
$$

Then $0 \in D\left(T_{1}\right)$, and $T_{1}$ and $T_{2}$ satisfy the hypothesis of Theorem 1 of [6], as is easily verified. This implies that $R\left(T_{1}+T_{2}\right)$ is all of $X^{*}$, and of course $R\left(T_{1}+T_{2}\right)$ is the same as $R(T+\lambda J)$. Also, $T+\lambda J$ is a maximal monotone operator by Theorem 2 of [6] $\left(^{2}\right)$. Thus $(T+\lambda J)^{-1}$ is a maximal monotone operator from $X^{*}$ to $X$ whose effective domain is all of $X^{*}$. We shall show that $(T+\lambda J)^{-1}$ is single-valued, and this will imply by Rockafellar [14, Corollary 1 to Theorem 1] that $(T+\lambda J)^{-1}$ is demicontinuous. Let $x$ and $y$ be elements of $(T+\lambda J)^{-1}\left(u^{*}\right)$, and let

$$
x^{*}=u^{*}-\lambda J(x) \in T(x), \quad y^{*}=u^{*}-\lambda J(y) \in T(y) .
$$

Since $T$ is monotone, we have

$$
\begin{aligned}
0 & =\left\langle x-y, u^{*}-u^{*}\right\rangle=\left\langle x-y,\left(x^{*}+\lambda J(x)\right)-\left(y^{*}+\lambda J(Y)\right)\right\rangle \\
& =\left\langle x-y, x^{*}-y^{*}\right\rangle+\lambda\langle x-y, J(x)-J(y)\rangle \\
& \geqq \lambda\langle x-y, J(x)-J(y)\rangle,
\end{aligned}
$$

and this implies $x=y$ by (2.2).

COROllary. Let $X$ be reflexive, and let $J$ be the duality mapping defined above. Let $T: X \rightarrow X^{*}$ be a monotone operator. In order that $T$ be maximal, it is necessary and sufficient that $R(T+J)$ be all of $X^{*}$.

Proof. By Zorn's Lemma, there exists a maximal monotone operator $T^{\prime}: X \rightarrow X^{*}$ such that $T^{\prime}(x) \supset T(x)$ for every $x$. Applying Proposition 1 to $T^{\prime}$ with $\lambda=1$, one sees that, for each $u^{*} \in X^{*}$, there exist unique elements $x \in X$ and $x^{*} \in X^{*}$ such that $x^{*} \in T^{\prime}(x)$ and $x^{*}+J(x)=u^{*}$. Therefore, $T=T^{\prime}$ if and only if each $u^{*} \in X^{*}$ can actually be expressed in the form $x^{*}+J(x)$ for some $x \in X$ and $x^{*} \in T(x)$, i.e. if and only if $R(T+J)=X^{*}$.

The next result may be regarded as a generalization of Theorem 2.2 of Browder [3]. Its proof is derived from Browder's proof of Theorem 3 of [6].

Proposition 2. Let $X$ be reflexive, and let $T: X \rightarrow X^{*}$ be a maximal monotone operator. Suppose there exists an $\alpha>0$ such that

$$
\left\langle x, x^{*}\right\rangle \geqq 0 \quad \text { whenever }\|x\|>\alpha, x \in D(T), x^{*} \in T(x) .
$$

(2) The hypothesis in Theorem 1 of [6] that $T_{1}$ have a dense domain is superfluous-it is nowhere used in the proof nor mentioned in the rest of the paper. 
Then there exists an $x \in X$ such that

$$
0 \in T(x) .
$$

Proof. We can assume that the given norm on $X$ has the special properties described above. Let $U_{\alpha}$ be the closed ball of radius $\alpha$ around the origin of $X$. The set

$$
T\left(U_{\alpha}\right)=\bigcup\{T(x) \mid\|x\| \leqq \alpha\}
$$

is closed in $X^{*}$; see Rockafellar [14, Lemma 2]. Hence, to show that $0 \in T\left(U_{\alpha}\right)$, it suffices to show that $T\left(U_{\alpha}\right)$ meets every ball of positive radius about the origin of $X^{*}$. Let $J$ be the duality mapping defined above. Given any $\varepsilon>0$, there exists by Proposition 1 an $x \in X$ such that

$$
0 \in(T+(\varepsilon / \alpha) J)(x) .
$$

Setting $x^{*}=-(\varepsilon / \alpha) J(x)$, we have $x^{*} \in T(x)$ and

$$
0=\left\langle x, x^{*}\right\rangle+(\varepsilon / \alpha)\langle x, J(x)\rangle,
$$

or equivalently by $(2.1)$

$$
\left\langle x, x^{*}\right\rangle=-(\varepsilon / \alpha)\|x\|^{2} .
$$

If we had $\|x\|>\alpha$, the left side of (2.8) would be nonnegative by (2.5), whereas the right side would be negative, a contradiction. Therefore $\|x\| \leqq \alpha$ and

$$
\left\|x^{*}\right\|=(\varepsilon / \alpha)\|J(x)\|=(\varepsilon / \alpha)\|x\| \leqq \varepsilon .
$$

This shows that $T\left(U_{\alpha}\right)$ meets the closed ball of radius $\varepsilon$ about the origin of $X^{*}$, and the proof is complete.

Besides the important device of perturbing a monotone operator $T$ to an operator of the form $T+\lambda J$, we shall need to use a device of "truncation" in proving Theorem 1. For each $\alpha>0$, we shall denote by $B_{\alpha}$ the subdifferential of the indicator function of the closed ball $U_{\alpha}$ of radius $\alpha$ about the origin in $X$ (with respect to a norm on $X$ having the special properties described above). Thus $B_{\alpha}$ is the normality operator for $U_{\alpha}$, so that $B_{\alpha}(x)=\varnothing$ when $\|x\|>\alpha, B_{\alpha}(x)$ consists of solely the zero element of $X^{*}$ when $\|x\|<\alpha$, and

$$
B_{\alpha}(x)=\{\lambda J(x) \mid \lambda \geqq 0\} \text { when }\|x\|=\alpha .
$$

It is known that $B_{\alpha}$ is a maximal monotone operator (Rockafellar [12], [13]). In particular, therefore, if $T: X \rightarrow X^{*}$ is any monotone operator, $T+B_{\alpha}$ is a monotone operator. Note that

$$
\begin{gathered}
\left(T+B_{\alpha}\right)(x)=T(x) \text { when } x \in \text { int } U_{\alpha}, \\
D\left(T+B_{\alpha}\right)=D(T) \cap U_{\alpha} .
\end{gathered}
$$

Proposition 3. Let $X$ be reflexive, and let $T: X \rightarrow X^{*}$ be a monotone operator 
such that $0 \in D(T)$. Suppose there exists an $\alpha_{0}>0$ such that the monotone operator $T+B_{\alpha}$ is maximal for every $\alpha \geqq \alpha_{0}$, where $B_{\alpha}$ is the mapping defined above. Then $T$ is maximal.

Proof. Subtracting a constant mapping from $T$ if necessary, we can reduce to the case where $0 \in T(0)$. Let $J$ be the duality mapping defined above, and let $u^{*}$ be an arbitrary element of $X^{*}$. To prove that $T$ is maximal, it suffices, according to the corollary to Proposition 1, to prove the existence of an $x \in X$ such that

$$
u^{*} \in(T+J)(x) \text {. }
$$

Take any $\alpha \geqq \alpha_{0}$ such that $\alpha>\left\|u^{*}\right\|$. Since $T+B_{\alpha}$ is maximal by hypothesis, there exists by Proposition 1 a certain $x \in X$ such that

$$
u^{*} \in\left(T+B_{\alpha}+J\right)(x)=(T+J)(x)+B_{\alpha}(x) .
$$

By the definition of $B_{\alpha}$, this means that

$$
u^{*} \in(T+J)(x)+\lambda J(x)=(T+(1+\lambda) J)(x),
$$

where $\|x\| \leqq \alpha$, and $\lambda$ is some nonnegative number, taken to be 0 if $\|x\|<\alpha$. We shall show that indeed $\|x\|<\alpha$, so that (2.12) reduces to (2.11) as desired. According to (2.12), there exists an $x^{*} \in T(x)$ such that

$$
u^{*}=x^{*}+(1+\lambda) J(x) \text {. }
$$

In terms of this $x^{*}$, we have

$$
\left\langle x, u^{*}\right\rangle=\left\langle x, x^{*}\right\rangle+(1+\lambda)\langle x, J(x)\rangle,
$$

where $\left\langle x, x^{*}\right\rangle \geqq 0$ by the monotonicity of $T$ and the fact that $0 \in T(0)$. It follows by (2.1) that

$$
(1+\lambda)\|x\|^{2}=(1+\lambda)\langle x, J(x)\rangle \leqq\left\langle x, u^{*}\right\rangle \leqq\|x\| \cdot\left\|u^{*}\right\|,
$$

and hence that

$$
\|x\| \leqq(1+\lambda)^{-1}\left\|u^{*}\right\|<(1+\lambda)^{-1} \alpha \leqq \alpha .
$$

\section{Proofs of the main results.}

Proof of Theorem 1. Since assumptions (a) and (b) in Theorem 1 are equivalent by [14, Theorem 1], as already pointed out, we need only consider the case of assumption (a). We shall suppose, to begin with, that $D\left(T_{2}\right)$ is also a bounded subset of $X$. Subsequently we shall prove, using Proposition 3 , that this boundedness assumption is unnecessary. It will be assumed, of course, that the norm on $X$ which we work with has the special properties described at the beginning of $\$ 2$.

Translating $T_{1}$ and $T_{2}$ by a common amount if necessary, and subtracting a constant mapping from $T_{1}$, we can assume that

$$
0 \in T_{1}(0), \quad 0 \in \text { int } D\left(T_{2}\right) .
$$


Let $J$ be the duality mapping defined above. We shall show that, for the monotone operator $T_{1}+T_{2}, R\left(T_{1}+T_{2}+J\right)$ is all of $X^{*}$, and this will establish that $T_{1}+T_{2}$ is maximal, according to the corollary to Proposition 1 .

Given any $\bar{x}^{*} \in X^{*}$, we must show that $\bar{x}^{*} \in R\left(T_{1}+T_{2}+J\right)$. Subtracting a constant mapping from $T_{2}$ if necessary, we can reduce the argument to the case where $\bar{x}^{*}=0$. Thus we need only show the existence of an $x \in X$ such that

$$
0 \in\left(T_{1}+T_{2}+J\right)(x) \text {. }
$$

Now $x$ satisfies (3.2) if and only if there exists an $x^{*} \in X^{*}$ such that

$$
-x^{*} \in\left(T_{1}+(1 / 2) J\right)(x) \text { and } x^{*} \in\left(T_{2}+(1 / 2) J\right)(x) \text {. }
$$

Define the mappings $S_{1}$ and $S_{2}$ from $X^{*}$ to $X$ by

$$
\begin{aligned}
& S_{1}\left(x^{*}\right)=-\left(T_{1}+(1 / 2) J\right)^{-1}\left(-x^{*}\right), \\
& S_{2}\left(x^{*}\right)=\left(T_{2}+(1 / 2) J\right)^{-1}\left(x^{*}\right) .
\end{aligned}
$$

The existence of an $x$ and $x^{*}$ satisfying (3.3) is then equivalent to the existence of an $x^{*}$ satisfying

$$
0 \in S_{1}\left(x^{*}\right)+S_{2}\left(x^{*}\right) .
$$

Therefore, to prove the existence of an $x$ satisfying (3.2), it suffices to prove that

$$
0 \in R\left(S_{1}+S_{2}\right) \text {. }
$$

To do this, we observe from Proposition 1 that $S_{1}$ and $S_{2}$ are single-valued maximal monotone operators, continuous from the strong topology of $X^{*}$ to the weak topology of $X$, such that

$$
D\left(S_{1}\right)=X^{*}=D\left(S_{2}\right) .
$$

Hence $S_{1}+S_{2}$ is a single-valued monotone operator, continuous from the strong topology to the weak topology, such that $D\left(S_{1}+S_{2}\right)=X^{*}$, and this implies by Browder [3, Theorem 1.2] that $S_{1}+S_{2}$ is maximal. Since $J(0)=0$, we have

$$
0 \in\left(T_{1}+(1 / 2) J\right)(0)
$$

by (3.1), and consequently $0 \in S_{1}(0)$. Therefore

$$
\left\langle S_{1}\left(x^{*}\right), x^{*}\right\rangle \geqq 0, \quad \forall x^{*} \in X^{*},
$$

by the monotonicity of $S_{1}$. Furthermore, $R\left(S_{2}\right)=D\left(T_{2}+(1 / 2) J\right)=D\left(T_{2}\right)$, so that $R\left(S_{2}\right)$ is a bounded set by our initial assumption and, by (3.1),

$$
0 \in \text { int } R\left(S_{2}\right) \text {. }
$$

We shall show that these properties of $R\left(S_{2}\right)$ imply the existence of an $\alpha>0$ such that

$$
\left\langle S_{2}\left(x^{*}\right), x^{*}\right\rangle \geqq 0 \quad \text { whenever }\left\|x^{*}\right\|>\alpha
$$


This will establish (3.7) as desired, via Proposition 2, since by (3.10) and (3.12) we will have

$$
\left\langle\left(S_{1}+S_{2}\right)\left(x^{*}\right), x^{*}\right\rangle \geqq 0 \text { whenever }\left\|x^{*}\right\|>\alpha .
$$

The proof that (3.12) holds for some $\alpha>0$ proceeds as follows. For any $x^{*}$ and $y^{*}$ in $X^{*}$, we have

$$
\left\langle S_{2}\left(x^{*}\right)-S_{2}\left(y^{*}\right), x^{*}-y^{*}\right\rangle \geqq 0
$$

by the monotonicity of $S_{2}$, in other words

$$
\left\langle S_{2}\left(x^{*}\right), x^{*}\right\rangle \geqq\left\langle S_{2}\left(y^{*}\right), x^{*}\right\rangle+\left\langle S_{2}\left(x^{*}\right)-S_{2}\left(y^{*}\right), y^{*}\right\rangle .
$$

Since $R\left(S_{2}\right)$ is bounded in $X, R\left(S_{2}\right)$ is contained in a certain ball of radius $\alpha_{1}>0$ about the origin, and hence

$$
\left|\left\langle S_{2}\left(x^{*}\right)-S_{2}\left(y^{*}\right), y^{*}\right\rangle\right| \leqq 2 \alpha_{1}\left\|y^{*}\right\|
$$

in (3.13). On the other hand, (3.11) implies by [14, Theorem 1] that $S_{2}^{-1}$ is locally bounded at 0 . Thus there exist $\varepsilon>0$ and $\alpha_{2}>0$ such that

$$
\left\{S_{2}\left(y^{*}\right) \mid\left\|y^{*}\right\| \leqq \alpha_{2}\right\} \supset\{y \mid\|y\| \leqq \varepsilon\} .
$$

From (3.13) and (3.14), we have

$$
\left\langle S_{2}\left(x^{*}\right), x^{*}\right\rangle \geqq\left\langle S_{2}\left(y^{*}\right), x^{*}\right\rangle-2 \alpha_{1} \alpha_{2}
$$

for every $y^{*}$ with $\left\|y^{*}\right\| \leqq \alpha_{2}$, so that by (3.15)

$$
\begin{aligned}
\left\langle S_{2}\left(x^{*}\right), x^{*}\right\rangle & \geqq \sup _{\|y\| \geqq \varepsilon}\left\{\left\langle y, x^{*}\right\rangle-2 \alpha_{1} \alpha_{2}\right\} \\
& =\varepsilon\left\|x^{*}\right\|-2 \alpha_{1} \alpha_{2} .
\end{aligned}
$$

The latter expression is nonnegative when $\left\|x^{*}\right\| \geqq 2 \alpha_{1} \alpha_{2} / \varepsilon$, and therefore (3.12) holds, as claimed, for $\alpha \geqq 2 \alpha_{1} \alpha_{2} / \varepsilon$.

The argument that we have given so far proves that Theorem 1 is valid under the additional hypothesis that $D\left(T_{2}\right)$ is bounded. We shall show now that this narrower version of Theorem 1 implies the general version. Let $T_{1}$ and $T_{2}$ be maximal monotone operators such that

$$
D\left(T_{1}\right) \cap \operatorname{int} D\left(T_{2}\right) \neq \varnothing,
$$

where $D\left(T_{2}\right)$ is not necessarily bounded. Translating the domains of $T_{1}$ and $T_{2}$ if necessary, we can assume that the origin belongs to the intersection (3.16). For each $\alpha>0$, the maximal monotone mapping $B_{\alpha}$ described in $\S 2$ then satisfies $D\left(T_{2}\right) \cap$ int $D\left(B_{\alpha}\right) \neq \varnothing$, and $D\left(B_{\alpha}\right)$ is bounded. The monotone operator $T_{2}+B_{\alpha}$ is therefore maximal by the narrower version of Theorem 1 . Since

$$
D\left(T_{2}+B_{\alpha}\right)=\left\{x \in D\left(T_{2}\right) \mid\|x\| \leqq \alpha\right\},
$$

and the origin belongs to the intersection (3.16), we have $D\left(T_{1}\right) \cap \operatorname{int} D\left(T_{2}+B_{\alpha}\right)$ 
$\neq \varnothing$, where $D\left(T_{2}+B_{\alpha}\right)$ is again bounded. Thus the mapping $T_{1}+\left(T_{2}+B_{\alpha}\right)=$ $\left(T_{1}+T_{2}\right)+B_{\alpha}$ is a maximal monotone operator for every $\alpha>0$ by the narrower version of Theorem 1, and we may conclude from Proposition 3 that $T_{1}+T_{2}$ is maximal. This proves Theorem 1 in the general case.

Proof of Theorem 2. Translating $T_{1}$ and $T_{2}$ by a common amount if necessary, we can suppose that

$$
0 \in \text { ri } D\left(T_{1}\right) \cap \text { ri } D\left(T_{2}\right),
$$

so that the affine hulls of $D\left(T_{1}\right)$ and $D\left(T_{2}\right)$ are certain subspaces $L_{1}$ and $L_{2}$ of $X$, respectively. Let $L_{0}=L_{1} \cap L_{2}$, and for $i=0,1,2$ let

$$
\begin{aligned}
& L_{i}^{\perp}=\left\{x^{*} \in X^{*} \mid\left\langle x, x^{*}\right\rangle=0, \forall x \in L_{i}\right\}, \\
& P_{i}(x)=L_{i}^{\perp} \text { if } x \in L_{i}, \\
&=\varnothing \text { if } x \notin L_{i} .
\end{aligned}
$$

Each $P_{i}$ is a maximal monotone operator (the subdifferential of the indicator of $L_{i}$ ), and

$$
P_{0}=P_{1}+P_{2}=P_{0}+P_{1}=P_{0}+P_{2} \text {. }
$$

Given any $x \in D\left(T_{1}\right), x^{*} \in T_{1}(x), z^{*} \in L_{1}^{\perp}$, we have $\left\langle x-y,\left(x^{*}+z^{*}\right)-y^{*}\right\rangle=$ $\left\langle x-y, x^{*}-y^{*}\right\rangle \geqq 0$ whenever $y \in D\left(T_{1}\right), y^{*} \in T(y)$, because $T_{1}$ is a monotone operator with $D\left(T_{1}\right) \subset L_{1}$, and this implies by the maximality of $T_{1}$ that

Thus

$$
x^{*}+z^{*} \in T_{1}(x) \text {. }
$$

$$
T_{1}=T_{1}+P_{1}
$$

Similarly

$$
T_{2}=T_{2}+P_{2}
$$

and it follows that

$$
T_{1}+T_{2}=T_{1}+T_{2}+P_{1}+P_{2}=\left(T_{1}+P_{0}\right)+T_{2}
$$

In view of (3.20) and (3.21), we can regard $T_{1}$ and $P_{0}$ in a natural way as maximal monotone operators from the space $L_{1}$ to the quotient space $X^{*} / L_{1}^{\perp}$, which may be identified with the dual $L_{1}^{*}$ of $L_{1}$. Theorem 1 is applicable to these mappings from $L_{1}$ to $L_{1}^{*}$, since by (3.17) the origin belongs to the intersection of $D\left(P_{0}\right)=L_{0}$ and the interior, relative to $L_{1}$, of $D\left(T_{1}\right)$. Thus $T_{1}+P_{0}$ must be a maximal monotone operator from $X$ to $X^{*}$. Now we apply a similar argument to the space $L_{2}$. Since (3.22) holds and $\left(T_{1}+P_{0}\right)+P_{2}=T_{1}+P_{0}$, we can regard $T_{1}+P_{0}$ and $T_{2}$ as maximal monotone operators from $L_{2}$ to $X^{*} / L_{2}^{\frac{1}{2}}$, which may be identified with $L_{2}^{*}$. The interior of $D\left(T_{2}\right)$ relative to $L_{2}$ meets $D\left(T_{1}+P_{0}\right)=D\left(T_{1}\right) \cap L_{2}$ by (3.17), so $\left(T_{1}+P_{0}\right)+T_{2}$ is maximal by Theorem 1 . This means by (3.23) that $T_{1}+T_{2}$ is a maximal monotone operator from $X$ to $X^{*}$. 
Proof of Theorem 3. As already mentioned in $\S 1, T_{1}$ is a maximal monotone operator, since it is the subdifferential of a certain closed proper convex function, namely the indicator of $K$ [12], [13]. Therefore $T_{1}+T_{2}$ is, at all events, a monotone operator. Let $y \in X$ and $y^{*} \in X^{*}$ be such that

$$
\left\langle x-y, x^{*}-y^{*}\right\rangle \geqq 0 \quad \text { whenever } x^{*} \in T_{1}(x)+T_{2}(x),
$$

or in other words

$$
\begin{aligned}
& \left\langle x-y, x_{1}^{*}\right\rangle+\left\langle x-y, T_{2}(x)-y^{*}\right\rangle \geqq 0 \\
& \text { whenever } x \in K \text { and } x_{1}^{*} \text { is normal to } K \text { at } x .
\end{aligned}
$$

We shall demonstrate by a direct argument that

$$
y^{*} \in T_{1}(y)+T_{2}(y) \text {, i.e. } y^{*}-T_{2}(y) \in T_{1}(y),
$$

and this will prove that $T_{1}+T_{2}$ is maximal.

If $x$ is any point of $K$ and $x_{1}^{*}$ is normal to $K$ at $x$, then $\lambda x_{1}^{*}$ is likewise normal to $K$ at $x$ for every $\lambda \geqq 0$, so that by (3.25)

$$
\lambda\left\langle x-y, x_{1}^{*}\right\rangle+\left\langle x-y, T_{2}(x)-y^{*}\right\rangle \geqq 0, \quad \forall \lambda \geqq 0 .
$$

This implies that $\left\langle x-y, x_{1}^{*}\right\rangle \geqq 0$. Thus

$$
\left\langle x-y, x_{1}^{*}-0\right\rangle \geqq 0 \quad \text { whenever } x_{1}^{*} \in T_{1}(x),
$$

and since $T_{1}$ is a maximal monotone operator we may conclude that $0 \in T_{1}(y)$. Hence $y \in K$.

To complete the proof that (3.26) holds, i.e. that $y^{*}-T_{2}(y)$ is normal to $K$ at $y$, we need only show that

$$
\left\langle u-y, y^{*}-T_{2}(y)\right\rangle \leqq 0, \quad \forall u \in K .
$$

Fix any $u \in K$, and let

$$
x_{\lambda}=\lambda u+(1-\lambda) y, \quad 0<\lambda<1 .
$$

Since $y \in K$ and $K$ is convex, we have $x_{\lambda} \in K$. Therefore (3.25) holds for $x=x_{\lambda}$ and $x_{1}^{*}=0$, and we have

$$
\begin{aligned}
0 & \leqq\left\langle x_{\lambda}-y, T_{2}(x)-y^{*}\right\rangle=\lambda\left\langle u-y, T_{2}(x)-y^{*}\right\rangle \\
& =\lambda\left\langle u-y, T_{2}\left(x_{\lambda}\right)-T_{2}(y)\right\rangle-\lambda\left\langle u-y, y^{*}-T_{2}(y)\right\rangle .
\end{aligned}
$$

This implies that

$$
\left\langle u-y, y^{*}-T_{2}(y)\right\rangle \leqq\left\langle u-y, T_{2}\left(x_{\lambda}\right)-T_{2}(y)\right\rangle, \quad 0<\lambda<1 .
$$

Since $T_{2}$ is hemicontinuous, $T_{2}\left(x_{\lambda}\right)$ converges in the weak* topology to $T_{2}(y)$ as $\lambda$ decreases to 0 , and (3.27) must hold.

4. Applications. Theorems 1,2 and 3 may be used to get new theorems asserting that $R\left(T_{1}+T_{2}\right)$ is all of $X$, or that $0 \in R\left(T_{1}+T_{2}\right)$, and so forth. 
For example, Theorem 3 of Browder [6] leads to the following result, where a monotone operator $T: X \rightarrow X^{*}$ is said to be coercive if

$$
\lim _{\alpha \rightarrow+\infty}(1 / \alpha) \inf \left\{\left\langle x, x^{*}\right\rangle \mid x^{*} \in T(x),\|x\| \geqq \alpha\right\}=+\infty
$$

(inf $\varnothing$ being $+\infty$ by convention).

THEOREM 4. Let $X$ be reflexive, and let $T_{1}$ and $T_{2}$ be monotone operators satisfying the hypothesis of Theorem 1 (or Theorem 2 or Theorem 3), such that $T_{1}+T_{2}$ is coercive. Then $R\left(T_{1}+T_{2}\right)=X^{*}$.

(In particular, $T_{1}+T_{2}$ is coercive if $0 \in D\left(T_{1}\right)$ and $T_{2}$ is coercive, or vice versa. Also, $T_{1}+T_{2}$ is coercive trivially if $D\left(T_{1}\right) \cap D\left(T_{2}\right)$ is bounded.)

Proof. The monotone operator $T=T_{1}+T_{2}$ is maximal by Theorem 1 (or by Theorem 2 or Theorem 3 , as the case may be), and since $T$ is also coercive we have $R(T)=X^{*}$ according to Browder [6, Theorem 3]. (Browder's result assumes that the unit ball of $X^{*}$ is strictly convex and that $0 \in D(T)$. However, the strict convexity assumption can be avoided by giving the proof in terms of a duality mapping $J$ of the type employed in $\$ 2$. This is permissible, since passage to an equivalent norm on $X$ does not alter the coerciveness of the operator $T$. The assumption that $0 \in D(T)$ can then be avoided by invoking Proposition 1 of the present paper in the proof in place of Browder's Theorem 1.)

In the case where $T_{1}$ and $T_{2}$ satisfy the hypothesis of Theorem 3, Theorem 4 yields a result about variational inequalities proved independently by Browder [4] and Hartman-Stampacchia [8].

A condition for the existence of an $x$ satisfying

$$
0 \in T_{1}(x)+T_{2}(x)
$$

can be obtained at once by combining Theorems 1, 2 and 3 with Proposition 2 . In particular, taking $T_{1}$ or $T_{2}$ to be the normality operator associated with a convex set $K$, one obtains the following existence theorem for solutions to variational inequalities.

TheOREM 5. Let $X$ be reflexive, let $K$ be a closed convex subset of $X$, and let $A: X \rightarrow X^{*}$ be a (possibly multivalued) monotone operator. Suppose there exist an $a \in K$ and an $\alpha>0$ such that

$$
\left\langle x-a, x^{*}\right\rangle \geqq 0 \quad \text { whenever } x \in D(A) \cap K,\|x\|>\alpha, x^{*} \in A(x) .
$$

Suppose also that one of the following five conditions is satisfied:

(a) $D(A) \supset K$ and $A$ is single-valued and hemicontinuous on $K$, or

(b) $A$ is maximal and $K \cap$ int $D(A) \neq \varnothing$, or

(c) $A$ is maximal and $D(A) \cap$ int $K \neq \varnothing$, or

(d) $A$ is locally bounded at some $x \in K \cap \mathrm{cl} D(A)$ and maximal, or

(e) $X$ is finite-dimensional, $A$ is maximal and

$$
\text { ri } D(A) \cap \text { ri } K \neq \varnothing .
$$


Then the variational inequality for $A$ and $K$ has a solution, i.e. there exists at least one $x \in D(A) \cap K$ such that, for some $x^{*} \in A(x),-x^{*}$ is normal to $K$ at $x$.

Proof. Replacing $K$ and $A$ by the translates $K^{\prime}=K-a$ and $A^{\prime}(x)=A(x+a)$ if necessary, we can reduce the theorem to the case where $a=0$. In terms of the normality operator $N$ for $K$, which has $D(N)=K$, the solutions to the variational inequality for $A$ and $K$ are simply the points $x$ such that

$$
0 \in N(x)+A(x)=(N+A)(x) .
$$

Such a solution exists by Proposition 2, if the monotone operator $T=N+A$ is maximal, since condition (2.5) for $T$ is equivalent to condition (4.3) on $K$ and $A$ (when $0=a \in K$ ). Each of the conditions (a), (b), (c), (d) and (e) is sufficient, in view of Theorems 1, 2 and 3, for $T$ to be maximal.

When the set $K$ is given by a system of convex inequality constraints,

$$
K=\left\{x \mid f_{i}(x) \leqq 0, i=1, \ldots, m\right\},
$$

the normality condition in Theorem 5 can sometimes be expressed in terms of the subdifferentials $\partial f_{i}$ of the convex functions $f_{i}$ and certain Lagrange multipliers. In particular, there is the following result.

Corollary. Let $X$ be reflexive, and let $T: X \rightarrow X^{*}$ be a maximal monotone operator. Let $f_{1}, \ldots, f_{m}$ be continuous real-valued convex functions on $X$. Suppose there exist an $a \in X$ and an $\alpha>0$ such that

$$
\begin{aligned}
& \qquad \in \mathbb{a}(T) \text { and } f_{i}(a)<0 \text { for } i=1, \ldots, m, \\
& \left\langle x-a, x^{*}\right\rangle \geqq 0 \quad \text { whenever } x \in D(T), \quad x^{*} \in T(x), \\
& \|x\|>\alpha \text { and } f_{i}(x) \leqq 0 \text { for } i=1, \ldots, m .
\end{aligned}
$$

Then there exist real numbers $\lambda_{1}, \ldots, \lambda_{m}$ (Lagrange multipliers) and an $x \in X$ such that

$$
\begin{gathered}
\lambda_{i} \geqq 0, \quad f_{i}(x) \leqq 0, \quad \lambda_{i} f_{i}(x)=0, \quad i=1, \ldots, m, \\
0 \in T(x)+\lambda_{1} \partial f_{1}(x)+\cdots+\lambda_{m} \partial f_{m}(x) .
\end{gathered}
$$

Proof. For $K$ as in (4.4), we have $a \in D(T) \cap$ int $K$ by (4.5), so that the hypothesis of Theorem 5 is satisfied under condition (c). The corollary then follows from the fact that (since the inequality system $f_{i}<0, i=1, \ldots, m$, can be satisfied) the normal cone to $K$ at a point $x \in K$ is the union of $\lambda_{1} \partial f_{1}(x)+\cdots+\lambda_{m} \partial f_{m}(x)$ over all coefficients $\lambda_{i}$ such that $\lambda_{i} \geqq 0$ for indices $i$ such that $f_{i}(x)=0$ and $\lambda_{i}=0$ for indices $i$ such that $f_{i}(x)<0$. For the proof of the latter fact, see Rockafellar [11, p. 86]. (The argument in [11] concerns the case where $f_{1}, \ldots, f_{m}$ are Gâteaux differentiable, but it is easily extended to the general case.)

REMARK. When certain of the functions $f_{i}$ are actually affine (i.e. linear-plus-aconstant), the corresponding conditions $f_{i}(a)<0$ in $(4.5)$ may be weakened to 
$f_{i}(a) \leqq 0$, provided that the condition $a \in D(T)$ is strengthened at the same time to $a \in$ int $D(T)$. This follows by the argument given in [11, p. 87]. With this modification, the corollary may be applied to cases where the definition of $K$ involves constraints of the form $\langle x, b\rangle=\beta$, where $b \in X^{*}$, since such a constraint can always be re-expressed as a pair of affine inequality constraints:

$$
f_{1}(x)=\langle x, b\rangle-\beta \leqq 0, \quad f_{2}(x)=\beta-\langle x, b\rangle \leqq 0 .
$$

Observe that, according to (4.8) the "solution" $x$ whose existence is asserted in the corollary is in particular a solution to:

$$
0 \in S(x), \text { where } S=T+\lambda_{1} \partial f_{1}+\cdots+\lambda_{m} \partial f_{m} .
$$

Moreover, $S$ is a maximal monotone operator by Theorem 1 , since the subdifferentials $\partial f_{i}$ are maximal monotone operators with $D\left(\partial f_{i}\right)=X$. (A nonnegative multiple of a maximal monotone operator with effective domain $X$ is trivially another maximal monotone operator.) In particular, suppose in the corollary that $T$ is single-valued on $D(T)$, and that each $f_{i}$ is actually Gâteaux differentiable, so that the subdifferentials $\partial f_{i}$ reduce to single-valued gradient mappings $\nabla f_{i}$. Then $S$ is a single-valued maximal monotone operator with $D(S)=D(T)$, and (4.9) becomes an equation:

$$
0=S(x)=T(x)+\lambda_{1} \partial f_{1}(x)+\cdots+\lambda_{m} \partial f_{m}(x) .
$$

The Lagrange multipliers $\lambda_{i}$ thus make it possible sometimes to reduce variational inequalities to operator equations of a simpler sort, which can be useful of course in the analysis of the solutions $x$, at least in cases where $T$ is a differential or integral operator whose properties are well understood.

\section{REFERENCES}

1. E. Asplund, Averaged norms, Israel J. Math. 5 (1967), 227-233. MR 36 \#5660.

2. —- Positivity of duality mappings, Bull. Amer. Math. Soc. 73 (1967), 200-203. MR 34 \#6481.

3. F. E. Browder, Multi-valued monotone nonlinear mappings and duality mappings in Banach spaces, Trans. Amer. Math. Soc. 118 (1965), 338-351. MR 31 \#5114.

4. - Nonlinear monotone operators and convex sets in Banach spaces, Bull. Amer. Math. Soc. 71 (1965), 780-785. MR 31 \#5112.

5. —_, Problèmes nonlinéaires, Univ. of Montreal Press, Montreal, 1966.

6. - Nonlinear maximal monotone operators in Banach space, Math. Ann. 175 (1968), 89-113. MR 36 \#6989.

7. - Nonlinear variational inequalities and maximal monotone mappings in Banach spaces, Math. Ann. 185 (1970), 81-90.

8. P. Hartman and G. Stampacchia, On some non-linear elliptic differential-functional equations, Acta. Math. 115 (1966), 271-310. MR 34 \#6355.

9. C. Lescarret, Cas d'addition des applications monotones maximales dans une espace de Hilbert, C. R. Acad. Sci. Paris 261 (1965), 1160-1163. MR 34 \#645.

10. G. J. Minty, Monotone (nonlinear) operators in Hilbert space, Duke Math. J. 29 (1962), 341-346. MR 29 \#6319. 
11. R. T. Rockafellar, Extension of Fenchel's duality theorem for convex functions, Duke Math. J. 33 (1966), 81-89. MR 32 \#4517.

12. - Characterization of the subdifferentials of convex functions, Pacific J. Math. 17 (1966), 497-510. MR 33 \#1769.

13. - On the maximal monotonicity of subdifferential mappings, Pacific J. Math. 33 (1970).

14. - Local boundedness of nonlinear montone operators, Michigan Math. J. 16 (1969), $397-407$.

UNIVERSITY OF WASHINGTON,

SeAtTLE, Washington 98105 